\section{New effort starts up to evaluate AIDS education in United States}

\section{San Francisco}

THE largest effort to date to study the effectiveness of AIDS education in preventing infection by the virus is being funded by the National Institute of Mental Health (NIMH) and the National Institute of Drug Abuse (NIDA) at three centres around the country. Those who have been calling out for more research into AIDS prevention will say that the initiative is long overdue.

Although public health officials agree that education programmes are virtually the only tool for combating the spread of HIV (human immunodeficiency virus) infection, evaluating these programmes is just getting under way. Stephen B. Hulley, director of the Center for AIDS Prevention Studies at the University of California at San Francisco (UCSF), says the new centres represent the "first sizeable efforts in the whole field" of research on AIDS education and prevention.

The UCSF centre is a collaboration between the university, the San Francisco Department of Public Health, and MIRA (Multicultural Inquiry and Research on AIDS), a group of researchers who study the impact of AIDS on minorities. Funding comes from a recently awarded $\$ 7.1$ million, four-year grant, in addition to the \$3-million, five-year grant that launched the centre last year.

Research at the UCSF centre will include improved epidemiological studies to learn the prevalence of high-risk behaviour, and research to test the effectiveness of educational programmes in reducing that behaviour. The centre's 50 investigators will focus on intravenous drug users and their families, gay and bisexual men, and ethnic minorities. The centre is also conducting a study of highrisk behaviours in Rwanda, Africa and an international exchange programme to help researchers from Africa and Latin America study AIDS prevention in their own countries.

Hulley says the MIRA researchers are integral to the centre's programme, a major aim of which is to investigate the factors behind the disproportionate representation of minorities among people with AIDS.

Black and Hispanic researchers will have better cultural understanding of their minority study subjects, says Hulley. MIRA and health department researchers will provide contact with existing AIDS education programmes, facilitating the rapid translation of research results into more effective programmes and policy.

A second centre is Columbia University's HIV Center for Clinical and Behavioral Studies in New York, which received a \$19-million award last month. The New York Psychiatric Institute and Columbia Presbyterian Hospital are partners with Columbia University in the centre, and other regional hospitals will participate in the centre's activities.

Three of the five studies proposed by the centre will analyse the effectiveness of AIDS prevention education, with the emphasis on how best to reach adolescents, said Claudia Bial, spokeswoman for the centre.

Although members of the New York health department will not participate directly in the research, Bial says a close cooperation with the city will allow the researchers access to the adolescent high school students, runaways and sexoffenders who will be the subjects of the studies.

A third AIDS centre at the University of Miami School of Medicine received a \$5.5-million, five-year grant from NIMH and NIDA in November 1986. Called the Biopsychosocial Center for the Study of AIDS, the Miami centre is focusing its attention on the development of AIDS dementia and on how social and psychological factors influence the course of HIV infection.

Marcia Barinaga
THE picture shows the Roque de los Muchachos observatory on the Canary Island of La Palma, home of the William Herschel telescope, which recently started yielding its first images. The telescope, which took four years to build at a cost of $£ 12$ million, is run by Britain's Royal

\title{
Fewer projects funded in West Germany \\ Munich
}

THE interdisciplinary West German research programmes called Schwerpunktprogramme have been reduced this year, reflecting the difficult research funding situation.The Deutsche Forschungsgemeinschaft (DFG) decided in Bonn on 15 October that it would fund only eleven out of twenty-four applicants, down from the usual acceptance rate of two-thirds. The programmes run for five years and support research projects carried on in several laboratories at once, with yearly meetings among all involved researchers.

Schwerpunktprogramme account for almost 20 per cent of the research support given by the DFG. This year's new programmes focus on biosciences. Included are projects titled "Molecular and immunological mechanisms of hostparasite interactions" and "Chemical ecology". Projects were also approved in humanities and social sciences, natural sciences and engineering. The 110 Schwerpunktprogramme funded by the DFG will receive DM 188 million in 1988 . The overall budget has remained roughly constant since 1985 Steven Dickman

\section{First images from European telescope}

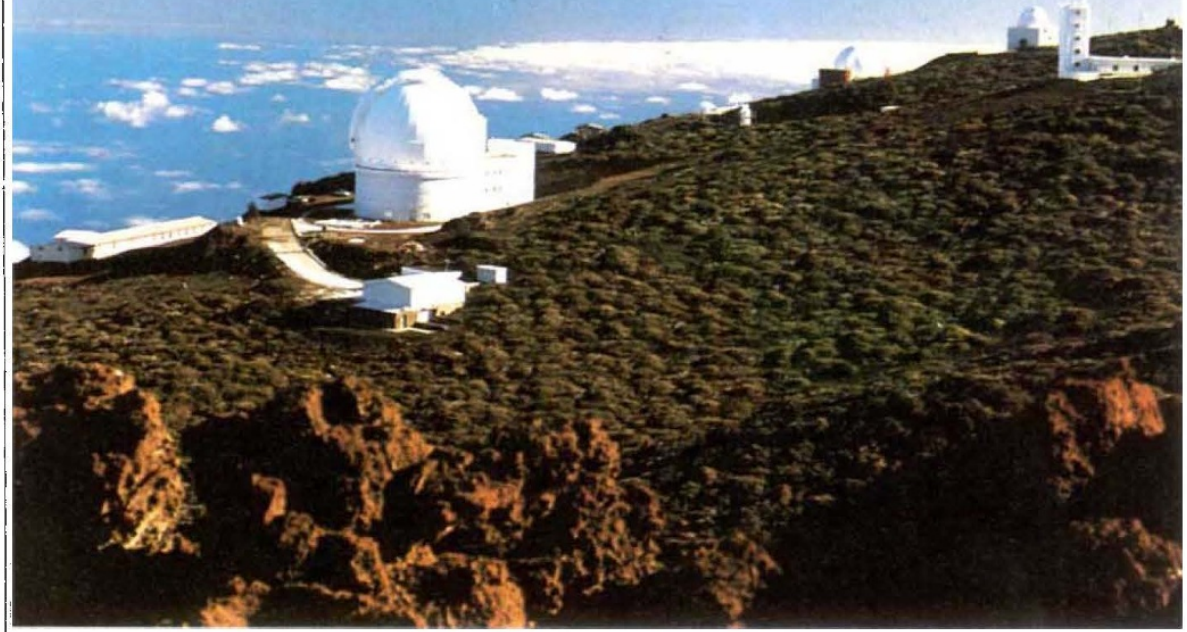

Greenwich Observatory in collaboration with the Netherlands Organization for the Advancement of Pure Research. It has a 4.2-metre mirror, making it the world's third largest single-mirror optical telescope after the US Palomar 5-metre and the Soviet 6-metre telescope. 